\title{
Prevalence and risk factors for low back pain among professional cooks working in school lunch services
}

\author{
Miwako Nagasu*1,2, Kazuhiro Sakai², Akiyoshi Ito ${ }^{3}$, Shigeru Tomita ${ }^{4}$, \\ Yoshiomi Temmyo ${ }^{5}$, Mitsuo Ueno ${ }^{6}$ and Shigeji Miyagi ${ }^{1}$
}

\begin{abstract}
Address: ${ }^{1}$ Department of Health Sciences, Kagawa Nutrition University, Saitama, Japan, ${ }^{2}$ The Institute for Science of Labour, Kanagawa, Japan, ${ }^{3}$ School of Health Sciences, University of Occupational and Environmental Health, Kitakyushu, Japan, ${ }^{4}$ School of Medicine, Dokkyo Medical University, Tochigi, Japan, ${ }^{5}$ Minatomachi Medical Center, Kanagawa, Japan and ${ }^{6}$ Institute of Occupational Safety and Health, All-Japan Prefectural and Municipal Workers' Union, Tokyo, Japan

Email: Miwako Nagasu* - minkykujira@hotmail.com; Kazuhiro Sakai - k.sakai@isl.or.jp; Akiyoshi Ito - ito-aki@health.uoeh-u.ac.jp; Shigeru Tomita - tomita07@nifty.com; Yoshiomi Temmyo - temmyo@cameo.plala.or.jp; Mitsuo Ueno - jdr74@ubcnet.or.jp;

Shigeji Miyagi - miyagi@eiyo.ac.jp

* Corresponding author
\end{abstract}

Published: 24 July 2007

BMC Public Health 2007, 7:17I doi:10.1 186/147|-2458-7-17|
Received: 20 December 2006

Accepted: 24 July 2007

This article is available from: http://www.biomedcentral.com/I47/-2458/7/I7/

(C) 2007 Nagasu et al; licensee BioMed Central Ltd.

This is an Open Access article distributed under the terms of the Creative Commons Attribution License (http://creativecommons.org/licenses/by/2.0), which permits unrestricted use, distribution, and reproduction in any medium, provided the original work is properly cited.

\begin{abstract}
Background: The prevalence of self-reported low back pain among professional cooks was estimated to examine the effects of daily life conditions, job-related factors, and psychological factors on this disorder.
\end{abstract}

Methods: Data was collected using a mailed self-administered questionnaire.

Results: Of 7100 cooks, 5835 (82\%) replied to the questionnaire, including 1010 men and 4825 women. The mean age was 41.4 for men and 47.5 for women. The prevalence of low back pain during a I-month period was $72.2 \%$ among men and $74.7 \%$ among women, with no significant differences between groups. By logistic regression analyses, factors significantly associated with the prevalence of low back pain in I month were female gender (prevalence ratio [PR] I.32; $95 \% \mathrm{Cl}$, I.03-I.68), current smoking (PR I.57; 95\% Cl, I.24-I.98), and past smoking (PR I.35; 95\% Cl, I.0II.79). As for job-related factors, the number of cooked lunches per person (PR I.28; $95 \% \mathrm{Cl}$, I.05I.56), breaks in the morning session (PR I.33; $95 \% \mathrm{Cl}$, I.13-1.56), kitchen environment (PR I.09; $95 \%, \mathrm{Cl}, \mathrm{I} .03-1.15$ ), and height of cooking equipment (PR I.I3; 95\% Cl, I.08-1.19) were associated with the prevalence of low back pain. As for psychological factors, job satisfaction (PR I.22; 95\% $\mathrm{Cl}, 1.03-1.45)$, stress at work (PR I.68; 95\% Cl, I.42-I.99), financial constraints (PR I.23;95\% Cl, I.03-I.47), health-related stress (PR I.31; 95\% Cl, I.08-1.59) and worries about the future (PR I.24; $95 \% \mathrm{Cl}, \mathrm{I} .0 \mathrm{I}-\mathrm{I} .52$ ) were similarly associated.

Conclusion: Daily life conditions, job-related factors, and psychological factors are associated with the occurrence of low back pain. It is important to take comprehensive preventive measures to address a range of work and life conditions that can be improved to decrease the incidence of low back pain for professional cooks. 


\section{Background}

Low back pain (LBP) is the most frequently reported work-related disorder in Japan [1]. Various studies [2,3] have reported that among working populations, the rate of LBP due to heavy workload during the previous day [4], week, month [5,6], or year [7-10], or during the lifetime $[4,9,11]$ varies between $5.6 \%$ and $84.1 \%$ [12].

LBP is a common disorder among cooks working in school lunch services in Japan [13-17]. Several studies have reported that the prevalence of back disorders during a specific period of employment ranged from $26.4 \%$ and $55.3 \%[14,16,18-20]$. The importance of studying risk factors that influence work-related disorders and the occurrence of LBP among the working population [21] is thus emphasized. Some studies report that female gender $[2,6,9,11,22-25]$ or higher age $[9,26]$ increase the risk of LBP, whereas other studies report no association between these factors $[6,9]$.

Recent studies have reported that psychological factors such as job satisfaction [27] and job strain [5,27] also may be risk factors for LBP. However, evidence for an association between psychological factors and LBP among cooks has not been studied in detail. The above-mentioned studies have found that demographic characteristics, daily life conditions, job-related factors, and psychological factors may influence the occurrence of LBP.

These studies indicate the need to take into account multiple work and life factors that can be changed to help reduce the prevalence of LBP, including daily life conditions (lifestyle and dietary habits), job-related factors (work activities and workplace environment), and psychological factors.

There have been reported interactions between the abovementioned factors. However, many earlier studies did not adjust for potential confounding variables. Therefore studies evaluating the risk factors associated with LBP are inconclusive. There is a lack of well-designed studies on the prevalence and risk factors associated with LBP among professional cooks, particularly those working in school lunch services, as they are exposed to high work loads [1317]. Therefore, we carried out this study on a nationally representative sample of cooks. The objectives of the study were (1) to estimate the prevalence of self-reported rates of LBP and (2) to identify factors that could influence the occurrence of LBP among cooks. Particular attention was paid to the physical kitchen environment as a potential cause of LBP. Attention was also paid to the links between LBP and psychological aspects of the work environment.

\section{Methods}

We conducted a cross-sectional survey using a mailed selfadministered questionnaire that investigated the prevalence of self-reported LBP and its association with various risk factors among professional cooks.

\section{Study setting and participants}

Participants included professional cooks working in school lunch services of the elementary schools of Japan. According to investigations, school lunches were provided at elementary schools all over Japan to about 7.19 million children in 2002 [28]. A total of 46,213 full-time cooks were working in the school lunch services in elementary schools and central kitchen systems in Japan during this period [29].

The principal task of these cooks was to provide meals for lunch for all pupils 5 days a week, year round, excluding seasonal vacation periods. There are two kinds of school lunch services in Japan. One of them consists of individual school kitchens in which lunch services are provided only for a particular school. The second type consists of services provided by medium kitchens and large kitchens, each of which delivers lunch service in large quantities to many schools.

The study sample was selected through the head office of the All-Japan Prefectural and Municipal Workers Union. There are a total of 47 prefectures in Japan. All prefectures and municipalities were included in our study to obtain proper representation of all of Japan. The union leaders maintained a list of the cooks working in school lunch services. A total of 500 cooks from 47 prefectures were selected. Similarly 200 cooks from each of 10 prefectures were selected (total 2000), 300 cooks from each of five prefectures (total 1500), and 100 cooks from each of 31 prefectures (total 3100) were randomly selected from a list of cooks. Thus a total of 7100 cooks were selected for this study.

\section{Questionnaire}

A mailed self-administered questionnaire was used to collect information about LBP and demographic characteristics, daily life conditions, job-related factors, and psychological factors. Prevalence of work-related disorders was ascertained by questionnaires used in previous studies on health hazards among cooks providing school lunch services $[19,20]$. This enabled us to compare the present study with previous ones. The questionnaire contained information about demographic factors such as age and gender. Daily life factors included information about lifestyle such as smoking, physical activities, and sleeping and eating habits. Information about job-related factors included work activities and conditions in the kitchens. Psychological factors included job satisfaction, stress at 
work, family-related stress, financial constraints, healthrelated problems, and worries about the future. Symptoms related to LBP, that is, episodes of pain, stiffness, or discomfort in the low back that were experienced during the previous 1 month and included in the questionnaire were adapted from a Japanese edition of a questionnaire used in previous studies $[19,20]$.

\section{Data collection}

A cross-sectional survey was carried out from September 2003 to February 2004. This study was supported by the All-Japan Prefectural and Municipal Workers Union. A questionnaire was mailed to 7100 professional cooks through the branches of the union located all over Japan. The questionnaire form was enclosed in an envelope that also contained an informed consent form. The anonymity and confidentiality of the information to be provided was detailed in the consent form. The participants were also free to refuse to respond to the questionnaire. The completed questionnaire forms were mailed back to the investigators.

For the purpose of this study, low back pain was defined as an experience of an episode or episodes of pain, stiffness, or discomfort of the low back anytime during the previous 1 month. The prevalence rate of LBP refers to the number of subjects who had reported at least period of LBP during the previous month.

\section{Data analysis}

The statistical analysis was carried out using the SPSS (12.0J Base System) computer package. The prevalence rates and their 95\% confidence intervals (CI) were calculated. Descriptive statistics were calculated for continuous variables. For continuous variables, the student t test was used to test the significance of observed differences. Observed differences between frequencies of categorical variables were tested using the chi square test.

We investigated the adjusted prevalence ratios (PR) and 95\% CIs of LBP by logistic regression analysis according to the following groups: gender, age, smoking habits, physical activity, sleeping habits, eating habits, work activities, environmental conditions in the kitchen, such as the kitchen environment score and the working height of cooking equipment score, job satisfaction, stress at work, family-related stress, financial constraints, health-related problems, and worries about the future.

The kitchen environment score and the working height of cooking equipment score were determined as follows: The kitchen environment score was estimated as the sum total of points indicating "no rest room," "wet floor system," "poor state of drainage," "presence of slippery places," "bumps and obstacles on the floor," and "noisy surround- ings." The working height of cooking equipment score was estimated as the number of different categories of cooking equipment having inappropriate heights, including counter-tables, kitchen sinks, cauldrons, vegetable cutters and peelers, feeding and finishing parts of tableware washing machines, and hot wind tableware dryers.

A generalized log linear model with binominal distribution was used to present associations between risk factors and LBP. PRs were estimatedas a measure of association. PRs were used because they are considered to be a better approximation of relative risk than odds ratio if the disease prevalence is high. Age was considered a potential confounding variable. Age was included in each logistic regression model, regardless of its association with LBP. A series of logistic regression models were used to identify the determinants of LBP. All self-reported risk factors were included in the regression models. PRs, 95\% CIs, and p values were calculated for each variable. A p value of 0.05 was considered statistically significant.

\section{Results}

Of 7100 cooks to whom the questionnaire was sent, 6,365 (90\%) returned the completed questionnaire forms by mail. Information about age and gender of respondents or other important information was incomplete on 530 forms. These replies were not included in the final analysis. Therefore 5835 replies were analyzed, for an overall response rate of $82 \%$.

Participants included 1010 men (17.3\%) and 4825 women $(82.7 \%)$. The mean \pm SD age of men $(41.4 \pm 9.8$ years) was significantly lower than that of women $(47.5 \pm$ 9.1 years $)(\mathrm{p}<0.001)$.

The overall 1-month prevalence of self-reported LBP was $74.3 \%$ among all the subjects. The prevalence of LBP among men and women was $72.2 \%$ and $74.7 \%$, respectively (Table 1 ). Differences by gender were not statistically significant. Differences in prevalence rates of LBP across different age groups were not statistically significant among men or among women.

We investigated the following risk factors among men and women: age, smoking habits, physical activities, sleeping habits, eating habits, work activities, workplace environment conditions in the kitchen, job satisfaction, stress at work, family-related stress, financial constraints, healthrelated problems, and worries about the future. Tables 2 , 3, 4 show prevalence rates of LBP according to the risk factors studied and also age-adjusted prevalence ratios of LBP stratified according to gender. As shown in Table 2, LBP was associated with daily life conditions such as smoking, physical activities, sleeping hours, and dietary habits among both men and women. 
Table I: Demographic characteristics of subjects

\begin{tabular}{|c|c|c|c|c|c|}
\hline & \multicolumn{5}{|c|}{ LBP in I month } \\
\hline & Total & Prevalence (\%) & Test ${ }^{1)}$ & PR non-adj 2) & $95 \% \mathrm{Cl}$ \\
\hline \multicolumn{6}{|l|}{ Gender } \\
\hline Male & 998 & 72.2 & & 1 & \\
\hline Female & 4781 & 74.7 & n.s & 1.13 & $0.97-1.32$ \\
\hline \multicolumn{6}{|l|}{ Age } \\
\hline$\leqq 39$ & 1400 & 75.4 & & I & \\
\hline $40 \sim 49$ & 1764 & 74.8 & n.s & 0.97 & $0.82-1.14$ \\
\hline $50 \leqq$ & 2615 & 73.3 & & 0.90 & $0.78-1.05$ \\
\hline \multicolumn{6}{|l|}{ Gender and age } \\
\hline \multicolumn{6}{|l|}{ Male } \\
\hline$\leqq 39$ & 428 & 73.8 & & 1 & \\
\hline $40 \sim 49$ & 337 & 73.0 & n.s & 0.96 & $0.69-1.32$ \\
\hline $50 \leqq$ & 233 & 68.2 & & 0.76 & $0.54-1.08$ \\
\hline \multicolumn{6}{|l|}{ Female } \\
\hline$\leqq 39$ & 972 & 76.0 & & 1 & \\
\hline $40 \sim 49$ & 1427 & 75.2 & n.s & 0.96 & $0.79-1.16$ \\
\hline $50 \leqq$ & 2382 & 73.8 & & 0.90 & $0.75-1.06$ \\
\hline
\end{tabular}

I) Chi-square test.

2) Prevalence ratio was not adjusted.

For women, regular physical activity (PR 1.27; 95\% CI, $1.07-1.50$ ), the number of hours of sleep (PR 1.30; 95\% $\mathrm{CI}, 1.14-1.49)$, and the number of cooked lunches per cook (150-199 meals; PR 1.53; 95\% CI, 1.30-1.79, 200 meals $\leqq$ PR $1.39 ; 95 \%$ CI, 1.17-1.65) were significantly associated with LBP. For men, none of these factors were associated with LBP; however, the irregularity of eating meals (PR 1.43; 95\% CI, 1.06-1.92) was significantly associated LBP among men.

For both sexes, current smoking habits (men; PR 1.59; 95\% CI, 1.15-2.02, women; PR 1.61; 95\% CI, 1.29-2.00), lack of breaks in the morning session (men; PR 1.50; 95\% CI, 1.13-2.01, women; PR 1.54; 95\% CI, 1.34-1.75), and working in large kitchens (men; PR 1.54; 95\% CI, 1.142.08, women; PR 1.27; 95\% CI, 1.08-1.60) were significantly associated with rates of LBP. Working environment factors significantly associated with rates of LBP for both sexes included noisy surroundings (men; PR 1.41; 95\% $\mathrm{CI}, 1.06-1.88$, women; PR 1.49; 95\% CI, 1.31-1.71), poor state of drainage (men; PR $1.43 ; 95 \% \mathrm{CI}, 1.06-1.92$, women; PR 1.29; 95\% CI, 1.23-1.46), and the presence of slippery places (men; PR 2.05; 95\% CI, 1.53-2.74, women; PR 1.60; 95\% CI, 1.40-1.83), bumps (men; PR

Table 2: Prevalence of self-reported low back pain and the associations with selected daily life conditions

\begin{tabular}{|c|c|c|c|c|c|c|c|c|c|c|}
\hline & \multicolumn{5}{|l|}{ Male } & \multicolumn{5}{|c|}{ Female } \\
\hline & Total & Prevalence(\%) & Pvaluel) & PR adj 2) & $95 \% \mathrm{Cl}$ & Total & Prevalence(\%) & Pvaluel) & PR adj 2) & $95 \% \mathrm{Cl}$ \\
\hline \multicolumn{11}{|l|}{ Smoking } \\
\hline Never & 260 & 65.4 & & I & & 3812 & 73.3 & & I & \\
\hline Ex-smokers & 199 & 72.4 & $*$ & 1.41 & $0.94-2.11$ & 305 & 78.4 & $* *$ & 1.31 & $0.98-1.73$ \\
\hline Current smokers & 523 & 75.1 & & 1.59 & $1.15-2.02$ & 598 & 81.6 & & 1.61 & $1.29-2.00$ \\
\hline \multicolumn{11}{|l|}{ Physical activity } \\
\hline Yes, regularly & 280 & 70.7 & & I & & 771 & 70.8 & & 1 & \\
\hline No & 718 & 72.8 & n.s & 1.15 & $0.84-1.56$ & 4010 & 75.4 & $* *$ & 1.27 & $1.07-1.50$ \\
\hline \multicolumn{11}{|l|}{ Sleeping hours } \\
\hline$\leqq 6$ hours & 553 & 71.6 & & 1 & & 3102 & 76.5 & & I & \\
\hline 7 hours $\leqq$ & 428 & 72.7 & n.s & 0.92 & $0.69-1.22$ & 1632 & 71.4 & ** & 1.30 & I.14-1.49 \\
\hline \multicolumn{11}{|l|}{ Having meals regularly } \\
\hline Regularly & 348 & 67.8 & & 1 & & 1949 & 73.5 & & I & \\
\hline Irregularly & 557 & 75.2 & $*$ & 1.43 & $1.06-1.92$ & 2636 & 75.5 & n.s & 1.11 & $0.97-1.26$ \\
\hline
\end{tabular}

1) Chi-square test was conducted for each item among men and among women. ${ }^{*} p<0.05$, ${ }^{* *} p<0.01$.

2) Prevalence ratio was adjusted for age group. 
Table 3: Prevalence of self-reported low back pain and the associations with selected job-related factors

\begin{tabular}{|c|c|c|c|c|c|c|c|c|c|c|}
\hline & \multicolumn{5}{|l|}{ Male } & \multicolumn{5}{|c|}{ Female } \\
\hline & Total & Prevalence(\%) & Pvaluel) & PR adj 2) & $95 \% \mathrm{Cl}$ & Total & Prevalence(\%) & Pvaluel) & PR adj 2) & $95 \% \mathrm{Cl}$ \\
\hline \multicolumn{11}{|l|}{ Work activities } \\
\hline \multicolumn{11}{|l|}{ Number of cooked lunches per cook } \\
\hline I-149 meals & 412 & 69.7 & & I & & 2457 & 71.2 & & I & \\
\hline 150-199 meals & 243 & 74.9 & n.s & 1.30 & $0.91-1.86$ & 1319 & 79.1 & **⿰冫⿰亅⿱丿丶丶 & 1.53 & $1.30-1.79$ \\
\hline 200 meals $\leqq$ & 340 & 73.2 & & 1.19 & $0.87-1.60$ & 985 & 77.5 & & 1.39 & $1.17-1.65$ \\
\hline \multicolumn{11}{|l|}{ Breaks in morning session } \\
\hline Yes & 549 & 68.9 & & 1 & & 2122 & 70.2 & & 1 & \\
\hline No & 426 & 77.0 & *** & 1.50 & $1.13-2.01$ & 2536 & 78.4 & *** & 1.54 & I.34-1.75 \\
\hline \multicolumn{11}{|l|}{ Type of workplace } \\
\hline Small kitchen & 314 & 66.6 & & 1 & & 3184 & 73.4 & & 1 & \\
\hline Medium kitchen $(<1500$ meals $)$ & 78 & 70.5 & $*$ & 1.20 & $0.70-2.06$ & 355 & 75.2 & $*$ & 1.10 & $0.85-1.42$ \\
\hline Large kitchen (1500 meals $\leqq)$ & 606 & 75.4 & & 1.54 & $1.14-2.08$ & 1242 & 77.8 & & 1.27 & $1.08-1.60$ \\
\hline \multicolumn{11}{|l|}{ Workplace environment factors } \\
\hline \multicolumn{11}{|l|}{ Kitchen environment } \\
\hline \multicolumn{11}{|l|}{ State of drainage } \\
\hline Good & 304 & 67.1 & & I & & 1760 & 71.5 & & 1 & \\
\hline Poor & 684 & 74.6 & $*$ & 1.43 & $1.06-1.92$ & 2907 & 76.5 & $* *$ & 1.29 & $1.23-1.46$ \\
\hline \multicolumn{11}{|l|}{ Slippery places on the floor } \\
\hline No & 340 & 62.6 & & I & & 2267 & 69.9 & & 1 & \\
\hline Yes & 637 & 77.7 & $* *$ & 2.05 & $1.53-2.74$ & 2399 & 78.9 & *** & 1.60 & $1.40-1.83$ \\
\hline \multicolumn{11}{|l|}{ Bumps on the floor } \\
\hline No & 598 & 68.7 & & 1 & & 2872 & 72.1 & & 1 & \\
\hline Yes & 377 & 78.2 & ** & 1.62 & $1.02-2.19$ & 1810 & 78.4 & *** & 1.39 & $|.22-1.6|$ \\
\hline \multicolumn{11}{|l|}{ Obstacles on the floor } \\
\hline No & 565 & 69.0 & & 1 & & 3063 & 73.5 & & 1 & \\
\hline Yes & 412 & 77.2 & *** & 1.51 & $1.13-2.02$ & 1599 & 77.0 & * & 1.20 & $1.04-1.38$ \\
\hline \multicolumn{11}{|l|}{ Noise level } \\
\hline Quiet & 583 & 69.5 & & I & & 2438 & 71.1 & & I & \\
\hline Noisy & 404 & 76.0 & * & 1.41 & $1.06-1.88$ & 2249 & 78.6 & 粎 & 1.49 & $|.3|-1.7 \mid$ \\
\hline \multicolumn{11}{|l|}{ Height of cooking equipment } \\
\hline \multicolumn{11}{|l|}{ Counter-tables } \\
\hline Fit & 568 & 68.0 & & 1 & & 3467 & 72.3 & & 1 & \\
\hline High or low & 420 & 78.1 & $* *$ & 1.66 & $1.24-2.24$ & 1242 & 80.9 & $* *$ & 1.62 & $1.38-1.90$ \\
\hline \multicolumn{11}{|l|}{ Kitchen sinks } \\
\hline Fit & 392 & 67.3 & & 1 & & 2432 & 68.5 & & 1 & \\
\hline High or low & 603 & 75.5 & *** & 1.45 & $1.09-1.93$ & 2274 & 81.2 & *** & 2.00 & $1.74-2.29$ \\
\hline \multicolumn{11}{|l|}{ Cauldrons } \\
\hline Fit & 772 & 68.9 & & 1 & & 3717 & 73.0 & & 1 & \\
\hline High or low & 209 & 85.6 & ** & 2.65 & $1.75-4.03$ & 950 & 80.8 & ** & 1.55 & $1.30-1.85$ \\
\hline \multicolumn{11}{|l|}{ Peelers } \\
\hline Fit & 685 & 70.4 & & I & & 3191 & 72.1 & & 1 & \\
\hline High or low & 254 & 77.6 & * & 1.44 & $1.03-2.02$ & 1189 & 81.1 & *** & 1.64 & $1.39-1.94$ \\
\hline \multicolumn{11}{|c|}{ Tableware washing machines - feeding } \\
\hline Fit & 776 & 70.6 & & 1 & & 3329 & 73.8 & & 1 & \\
\hline High or low & 193 & 79.8 & * & 1.60 & $1.09-2.36$ & 1071 & 78.7 & *** & 1.31 & $1.11-1.55$ \\
\hline Tableware washing machines - finishing & & & & & & & & & & \\
\hline Fit & 694 & 69.2 & & 1 & & 3013 & 73.1 & & 1 & \\
\hline High or low & 271 & 81.2 & *** & 1.89 & $1.34-2.67$ & 1379 & 79.0 & *** & 1.38 & $1.19-1.61$ \\
\hline
\end{tabular}

1) Chi-square test was conducted among men and women for each item. $*_{p}<0.05$, $*^{*} p<0.0$ I.

2) Prevalence ratio was adjusted for age group.

1.62 ; 95\% CI, 1.02-2.19, women; PR 1.39; 95\% CI, $1.22-$ 1.61 ), and obstacles on the floor (men; PR 1.51; 95\% CI, 1.13-2.02, women; PR 1.20; 95\% CI, 1.04-1.38).

As for height of cooking equipment, several items with variable working heights were significantly associated with rates of LBP, including counter-tables (men; PR 1.66; 95\% CI, 1.24-2.24, women; PR 1.62; 95\% CI, 1.38-1.90), kitchen sinks (men; PR 1.45; 95\% CI, 1.09-1.93, women; PR 2.00; 95\% CI, 1.74-2.29), cauldrons (men; PR 2.65;
95\% CI, 1.75-4.03, women; PR 1.55; 95\% CI, 1.30-1.85) and peelers (men; PR 1.44; 95\% CI, 1.03-2.02, women; PR 1.64; 95\% CI, 1.39-1.94), feeding (men; PR 1.60; 95\% CI, 1.09-2.36, women; PR 1.31; 95\% CI, 1.11-1.55) and finishing (men; PR 1.89; 95\% CI, 1.34-2.67, women; PR $1.38 ; 95 \% \mathrm{CI}, 1.19-1.61)$ parts of tableware washing machines.

A number of psychological factors were significantly associated with the prevalence of LBP in both sexes, including 
Table 4: Prevalence of self-reported low back pain and the associations with psychological factors

\begin{tabular}{|c|c|c|c|c|c|c|c|c|c|c|}
\hline & \multicolumn{5}{|l|}{ Male } & \multicolumn{5}{|c|}{ Female } \\
\hline & Total & Prevalence(\%) & Pvaluel) & PR adj 2) & $95 \% \mathrm{Cl}$ & Total & Prevalence(\%) & Pvaluel) & PR adj 2) & $95 \% \mathrm{Cl}$ \\
\hline \multicolumn{11}{|l|}{ Job satisfaction } \\
\hline Satisfied & 438 & 63.9 & & I & & 2944 & 72.1 & & I & \\
\hline Dissatisfied & 533 & 78.6 & $* *$ & 2.08 & I.57-2.77 & 1719 & 79.4 & *** & 1.49 & $|.29-| .7 \mid$ \\
\hline \multicolumn{11}{|l|}{ Stress at work } \\
\hline Fine & 350 & 58.9 & & 1 & & 1683 & 64.5 & & 1 & \\
\hline Stressful & 647 & 79.6 & $* *$ & 2.71 & $2.03-3.61$ & 3069 & 80.3 & ** & 2.23 & $1.95-2.55$ \\
\hline \multicolumn{11}{|l|}{ Family-related stress } \\
\hline Fine & 578 & 67.3 & & 1 & & 2268 & 70.9 & & I & \\
\hline Stressful & 411 & 79.8 & $* *$ & 1.94 & $1.44-2.62$ & 2478 & 78.2 & *** & 1.47 & $1.23-1.67$ \\
\hline \multicolumn{11}{|l|}{ Financial constraints } \\
\hline Fine & 290 & 62.4 & & 1 & & 1812 & 68.3 & & 1 & \\
\hline Stressful & 707 & 76.4 & $* *$ & 1.91 & $1.42-2.57$ & 2933 & 78.6 & *** & 1.71 & $1.50-1.96$ \\
\hline \multicolumn{11}{|l|}{ Health-related problems } \\
\hline Fine & 421 & 63.2 & & 1 & & $|75|$ & 66.5 & & 1 & \\
\hline Stressful & 567 & 79.4 & $* *$ & 2.32 & $1.74-3.09$ & 3002 & 79.5 & $* *$ & 1.97 & $1.73-2.25$ \\
\hline \multicolumn{11}{|c|}{ Worries about the future } \\
\hline Fine & 290 & 62.1 & & 1 & & 1265 & 65.1 & & 1 & \\
\hline Stressful & 700 & 76.7 & *** & 2.01 & $1.49-2.69$ & 3475 & 78.1 & *** & 1.91 & $1.66-2.20$ \\
\hline
\end{tabular}

1) Chi-square test was conducted among men and women for each item. ${ }^{*} \mathrm{p}<0.05$, ${ }^{* *} \mathrm{p}<0.01$.

2) Prevalence ratio was adjusted for age group.

job satisfaction (men; PR 2.08; 95\% CI, 1.57-2.77, women; PR 1.49; 95\% CI, 1.29-1.71), stress at work (men; PR 2.71; 95\% CI, 2.03-3.61, women; PR 2.23; 95\% CI, 1.95-2.55), family-related stress (men; PR 1.94; 95\% CI, 1.44-2.62, women; PR 1.47; 95\% CI, 1.23-1.67), financial constraints (men; PR 1.91; 95\% CI, 1.42-2.57, women; PR 1.71; 95\% CI, 1.50-1.96), health-related problems (men; PR 2.32; 95\% CI, 1.74-3.09, women; PR $1.97 ; 95 \% \mathrm{CI}, 1.73-2.25)$, and worries about the future (men; PR 2.01; 95\% CI, 1.49-2.69, women; PR 1.91; 95\% CI, 1.66-2.20).

The results of the regression analyses confirmed the significance of the relationship between many of these factors and the prevalence of LBP, as shown in Table 5. Females (PR 1.32; 95\% CI, 1.03-1.68) as well as current (PR 1.57; 95\% CI, 1.24-1.98) and ex-smokers (PR 1.35; 95\% CI, 1.01-1.79) were more likely to suffer from LBP. Among work activities, cooking more lunches per person (PR 1.28; $95 \% \mathrm{CI}, 1.05-1.56)$ and a lack of breaks in the morning session (PR 1.33; 95\% CI, 1.13-1.56) were associated with LBP. In addition to these factors, the scores of kitchen environment (PR 1.09; 95\% CI, 1.03-1.15) and the working height of the cooking equipment (PR 1.13; 95\% CI, 1.08-1.19) were also associated with LBP. The median (interquartile range) of the kitchen environment score was 2 (1-4). The median (interquartile range) of the working height of cooking equipment score was 2 (1-3).
Among the psychological factors, job dissatisfaction (PR $1.22 ; 95 \% \mathrm{CI}, 1.03-1.45$ ), stress at work (PR 1.68; 95\% CI, 1.42-1.99), financial constraints (PR 1.23; 95\% CI, 1.03-1.47), health-related problems (PR 1.31; 95\% CI, 1.08-1.59), and worries about the future (PR 1.24; 95\% CI, 1.01-1.52) were significantly associated with the higher rates of LBP. It was noteworthy that the highest prevalence ratio was found for stress at work.

\section{Discussion}

In this cross-sectional study, the prevalence of selfreported LBP was high among professional cooks working in school lunch services. This high prevalence was noted regardless of age and gender. The questionnaire results indicated that the prevalence of LBP was associated with daily life conditions, job-related factors, and psychological factors. These results are important as the study was conducted in a large sample of both male and female cooks selected from all over Japan, with a good response rate of $82 \%$. This high response rate was possible through the inclusion of an informed consent form and the assurance of confidentiality of the information provided. The results may thus be considered representative of cooks engaged in public school lunch services.

It was of particular interest that $17.3 \%$ of our sample was male, considering that the proportion of male cooks in school lunch services is low $[16,17]$. Previous studies 
Table 5: Adjusted prevalence ratios of low back pain for each factor

\begin{tabular}{|c|c|c|c|}
\hline & & PR adjl) & $95 \% \mathrm{Cl}$ \\
\hline Gender & Female & 1.32 & $1.03-1.68$ \\
\hline \multirow[t]{2}{*}{ Smoking } & Ex-smokers & 1.35 & $1.01-1.79$ \\
\hline & Current smokers & 1.57 & $1.24-1.98$ \\
\hline $\begin{array}{l}\text { The number of cooked lunches } \\
\text { per person }\end{array}$ & $150-199$ meals & 1.28 & $1.05-1.56$ \\
\hline No breaks in morning session & & 1.33 & $1.13-1.56$ \\
\hline Kitchen environment score ${ }^{2}$ ) & & 1.09 & $1.03-1.15$ \\
\hline $\begin{array}{l}\text { The height of cooking equipment } \\
\text { score }{ }^{3)}\end{array}$ & & 1.13 & $1.08-1.19$ \\
\hline Job satisfaction & Dissatisfied & 1.22 & $1.03-1.45$ \\
\hline Stress at work & Stressful & 1.68 & $1.42-1.99$ \\
\hline Financial constraints & Stressful & 1.23 & $1.03-1.47$ \\
\hline Health-related stress & Stressful & 1.31 & $1.08-1.59$ \\
\hline Worries about the future & Stressful & 1.24 & $1.01-1.52$ \\
\hline
\end{tabular}

I) Prevalence ratios were adjusted for all items. Prevalence ratios referred to the following groups: male, age < 39 years, not smoking, doing physical activity, sleeping for less than 7 hours, having meals regularly, I-I49 meals per a cook, small-scale kitchen, having breaks in the morning session, and all psychological factors unstressful.

2) Kitchen conditions score is the sum total of the points such as no rest room, wet floor, poor state of drainage, presence of slippery places, bumps, and obstacles on the floor, and noisy surroundings (I point each).

3) The height of cooking equipment score is the sum total of the points such as the inappropriate height of counter-tables, kitchen sinks, cauldrons, vegetable cutters and peelers, feeding and finishing parts of tableware washing machines, and hot wind tableware dryers (I point each).

emphasized the importance of considering the health conditions of male and female cooks separately due to differences in their average height $[3,21]$.

One previous study reported that the prevalence of LBP was associated with stature among men [22]. Further studies may be necessary as this association of weight and tall stature with the occurrence of LBP was not reported in other studies $[3,25,30]$. As male and female cooks in our study shared the same kitchen and cooking equipment, the significant difference in the mean height of male cooks ( $169.0 \pm 6.60$ centimeters $)$ and that of female cooks (156.4 \pm 5.17 centimeters) was notable. To prevent LBP, it may be worth considering adjusting the working height of equipment in kitchens used for school lunch services to suit the male and female cooks.

\section{Prevalence of LBP}

The 1-month prevalence of LBP was $74.3 \%$ among all the subjects. This prevalence rate was higher than that reported in the previous studies $[5,20,31]$. The observed prevalence of LBP among cooks was higher than that in the general population (35\% and $42 \%$ among males and females, respectively) [6], staff members working in schools for physically and mentally handicapped children (45\%) [5], or physiotherapy students (44\%) [31]. The prevalence of LBP among cooks in our study was similar to the 1 -month prevalence ( $81 \%)$ reported by a previous study [32]. Kurumatani et al. reported that the rates of complaints related to the cervicobrachial area and low back were significantly higher in female cooks than in housewives of similar age [32]. Other previous studies also reported that the 1-month prevalence of LBP was $68 \%$ among cooks working in large kitchens [19] and $67 \%$ among cooks younger than 35 years [16]. The high prevalence of LBP among cooks reported by these studies may warrant the need to undertake preventive measures for improving health and work efficiency of professional cooks.

\section{Demographic characteristics}

According to our logistic regression analyses, LBP was associated with several risk factors. It was noteworthy that LBP was associated with gender after adjustment for all factors. The association of LBP with gender has also been reported by other studies $[3,24,26]$. LBP has also been shown to be more common among women than men in some occupations, such as white collar workers $[22,23,33]$, whereas it is more frequent for men than for women among peasant farmers [26]. We confirmed that among the professional cooks in Japan, the risk of LBP was higher among women than men.

In this study, age was not associated with the prevalence of LBP among cooks. Some studies have reported that the prevalence of LBP increases with age among farmers [26] and hospital staff [24]. Other studies have reported no associated between age and LBP [3].

\section{Daily life conditions}

We found a significant relation between smoking habits and the prevalence of LBP among cooks. Our study 
showed that cooks who were current and ex-smokers had a higher prevalence rate of LBP than those who had never smoked. After adjusting for all risk factors, current smokers had PR for LBP that was 1.5 times higher than that of non-smokers. Previous studies have reported that the incidence of LBP was higher among adolescents and working groups who had ever smoked [24]. The evidence for the association of smoking with LBP was also reported in studies on smoking and decreased bone mineral density in the lumber spine [34]. Gilgil et al. [9] reported that individuals who smoked 21 or more cigarettes per day for 15 years were more likely to suffer from LBP. The results of our study confirm the association of the occurrence of LBP with smoking habits.

Beija et al. reported that playing sports had a protective effect against LBP [24]. In our study, cooks who participated in regular physical activity reported a lower prevalence of LBP. Available literature about the association of LBP with regular sports/physical activity is inconclusive. Further research is needed to prove the effectiveness of sports/physical activity for preventing LBP among the working population.

After adjustment for age, the average duration of sleep was associated with LBP. Women cooks who slept for 7 hours or more per day reported a lower prevalence of LBP than women who slept for less than 7 hours. Generally, women slept for a shorter duration than men. This may be because women have additional responsibilities of housekeeping and childcare. Some studies indicate that housekeeping work and childcare could increase the risk of LBP among women [35-37].

\section{Job-related factors}

In our study, there was a clear association between jobrelated factors and LBP among cooks. Previous studies dealing with professional cooks also reported that jobrelated factors such as work activities, workplace conditions in the kitchen, and inappropriate cooking equipment increased the risk of LBP $[16,19,20,32,38]$.

Our study found that physical kitchen environment was variously associated with the prevalence of LBP. For example, cooks who took breaks in the morning session reported LBP less frequently than those who did not. A previous study reported that more frequent tasks involving materials handling, including working with heavy loads, during the morning hours than in the afternoon [14]. It has been suggested that introducing a period of rest in the morning session may have a positive influence on recovery from fatigue and reduction of symptoms such as pain and stiffness, especially in the back, neck and upper limbs among cooks $[14,16]$. It would be of interest to study the timing and duration of the break period during working hours.

It was found that the number of lunches cooked per person was associated with the occurrence of LBP. This was similar to results of a previous study in which the number of lunches cooked per person was associated with the rate of cervicobrachial complaints and LBP [32]. These findings suggest that the numbers of lunches cooked per person may relate to an increased workload, leading to a higher rate of LBP. A previous study [19] reported that the number of lunches cooked in large-scale kitchens was more than that in small-scale kitchens. Cooks who worked in large-scale kitchens were frequently assigned to carry of heavy objects. These heavier loads may relate to the more frequent occurrence of LBP and cervicobrachial symptoms $[17,22]$. These symptoms were less commonly reported by the cooks working in small-scale kitchens. However, in the multivariate model, cooks working in the biggest-scale kitchens were not shown to have an increased incidence of LBP.

The kitchen condition score in our study, which included the items such as noisy surroundings, poor state of drainage, and the presence of slippery places, bumps, and obstacles on the floor was associated LBP. In terms of noise in the working environment, a previous study reported that there were places in kitchens where $85 \mathrm{~dB}$ (A) was exceeded [14]. Measures against noise are thus necessary in kitchens.

In our study, working in kitchens with obstacles, including height differences, bumps, and slippery places was associated with a higher prevalence of LBP. This may be because pushcarts designed to reduce the need for lifting heavy loads cannot be used in such kitchens. A previous study [16] reported that handling of heavy materials affected the health of workers. A similar association was found between frequent manual handling of materials $[17,22]$ such as ingredients, tableware, and the prevalence of LBP among cooks $[21,39]$. These findings indicate the need to improve conditions of materials handling to help prevent LBP among cooks.

We found that the working height of cooking equipment, such as counter-tables, kitchen sinks, cauldrons, vegetable cutters, peelers, tableware washing machines, and tableware dryers was associated with the occurrence of LBP. These results were similar to those reported earlier that the risk of LBP increased if the height of cooking equipment did not suit the appropriate working height of the cooks [16,32]. According to Koda et al. [14], the workload of cooks was larger if they maintained uncomfortable standing postures, such as forward bending [16]. Poor working postures are thus known to increase the rate of LBP [40]. 
Training in working postures [40] and improvement of the working height of all types of cooking equipment may help reduce the rate of LBP.

\section{Psychological factors}

Psychological factors related to work, such as high work stress and low job satisfaction, have been linked with LBP in previous studies [27]. Nevertheless, the relationship between psychological factors and LBP among cooks has not been explored in detail. Our study confirmed a close association between psychological factors such as job satisfaction, stress at work, financial constraints, healthrelated problems, and worries about the future and the occurrence of LBP among cooks. In our study, higher rates of LBP were observed among the cooks who reported a higher level of stress. In a previous study, workers exposed to high stress at home and at work also suffered a significantly higher rate of LBP [40]. The need for training cooks to manage stress at the workplace and at home should be emphasized.

It may be vital to examine the health status of cooks in relation to their workplace conditions because it is reported that these conditions could cause not only LBP but also complaints in the neck, shoulders, and arms [18]. LBP is known to be a major reason for the loss of work days and therefore decreased productivity [12]. Many studies have confirmed that work-related injuries, disorders, and diseases can be prevented by proper risk assessment and employee training [41-44]. A need is suggested for conducting a longitudinal study to confirm the relationships observed in this study. Information on these relationships would be useful for developing training programs to prevent LBP among cooks.

\section{Conclusion}

The self-reported 1-month prevalence of LBP was high among professional cooks. This high prevalence was significantly associated with daily life conditions, job-related factors, and psychological factors. These results suggest the importance of taking comprehensive preventive measures. For reducing the prevalence of low back pain among cooks, it is generally necessary to address a range of work and life factors. Our results suggest the particular importance of improving the height of kitchen equipment, the physical environment of the kitchen, and other conditions relating to psychosocial aspects. It is useful to develop training programs for school managers and cooks aimed at the prevention of work-related disorders such as LBP.

\section{Abbreviations \\ LBP: Low back pain}

PRs: Prevalence Ratios

\section{Competing interests}

The author(s) declare that they have no competing interests.

\section{Authors' contributions}

$\mathrm{MN}$ : was the principal investigator, participated in the design and protocol preparation, participated in the data collection, data analysis and interpretation of results, and wrote and revised initial drafts of the manuscript.

KS: assisted in design and protocol preparation, and made a substantial contribution toward analysis, drafting the manuscript, and correcting the initial and final drafts of the manuscript.

AI: assisted in design and protocol preparation, made a substantial contribution to the analysis, drafting of manuscript and correcting the initial and final drafts of the manuscript.

ST: provided assistance with the analysis and manuscript preparation.

YT: contributed to the protocol, and helped with the design of the study and manuscript preparation.

MU: helped with the design, protocol, and data collection.

SM: conceived the study, set up the design, guided and participated in the analysis, and revised the final manuscript.

All authors read and approved the final manuscript for submission for publication.

\section{Acknowledgements}

We appreciate the support provided by Mr. Tomoyuki Hara and the professional cooks working in school lunch services of All-Japan Prefectural and Municipal Workers Union (JICHIRO). The authors thank Dr. Kazutaka Kogi of The Institute for Science of Labour, Kawasaki, Japan for his advice, technical assistance, and reading of the manuscript.

\section{References}

I. Japan Industrial Safety Health Association: General guidebook on industrial health (in Japanese) Tokyo, Japan: Japan Industrial Safety and Health Association; 2004

2. Shehab D, Al-Jarallah K, Moussa MA, Adham N: Prevalence of low back pain among physical therapists in Kuwait. Med Princ Pract 2003, I 2:224-230.

3. Burdorf A, Sorock G: Positive and negative evidence of risk factors for back disorders. Scand J Work Environ Health 1997, 23:243-256.

4. Jin K, Sorock GS, Courtney TK: Prevalence of low back pain in three occupational groups in Shanghai, People's Republic of China. J Safety Res 2004, 35:23-28.

5. Muto S, Muto T, Seo A, Yoshida T, Taoda K, Watanabe M: Prevalence of and risk factors for low back pain among staffs in schools for physically and mentally handicapped children. Ind Health 2006, 44:123-127. 
6. Papageorgiou AC, Croft PR, Ferry S, Jayson MI, Silman AJ: Estimating the prevalence of low back pain in the general population. Evidence from the South Manchester Back Pain Survey. Spine 1995, 20:1889-1894.

7. Mehling WE, Krause N: Are difficulties perceiving and expressing emotions associated with low-back pain? The relationship between lack of emotional awareness (alexithymia) and 12-month prevalence of low-back pain in I 180 urban public transit operators. J Psychosom Res 2005, 58:73-81.

8. Loney PL, Stratford PW: The prevalence of low back pain in adults: a methodological review of the literature. Phys Ther 1999, 79:384-396.

9. Gilgil E, Kacar C, Butun B, Tuncer T, Urhan S, Yildirim C, Sunbuloglu G, Arikan V, Tekeoglu I, Oksuz MC, Dundar U: Prevalence of low back pain in a developing urban setting. Spine 2005 30:1093-1098

10. Omokhodion FO, Umar US, Ogunnowo BE: Prevalence of low back pain among staff in a rural hospital in Nigeria. Occup Med (Lond) 2000, 50:107-I I0.

II. Cassidy JD, Carroll LI, Cote P: The Saskatchewan health and back pain survey. The prevalence of low back pain and related disability in Saskatchewan adults. Spine 1998, 23: I860- |866. discussion 1867

12. Lee P, Helewa A, Goldsmith CH, Smythe HA, Stitt LW: Low back pain: prevalence and risk factors in an industrial setting. J Rheumatol 200I, 28:346-35I.

13. Nagasu M: Prevalence and risk factors for work-related disorders in school lunch workers, Japan. In PhD thesis Kagawa Nutrition University, Health Sciences Department; 2005.

14. Koda S, Ogawa T, Kodera R, Oze Y, Aoyama H: An experimental study of fatigue symptoms and the effects of inserting rest periods in the work schedule of cooks providing school-lunch service (Abstract in English). Hirotokyuyounokagaku 1992, 7:5I-6I.

15. Nagira T, Suzuki J, Oze $\mathrm{Y}$, Ohara H, Aoyama H: Cervicobrachial and low-back disorders among school lunch workers and nursery-school teachers in comparison with cash-register operators. J Hum Ergol 198I, 10:1 I7-124.

16. Onishi N, Watanabe A, Sakai K, Shindo H, Tsukazaki Y, Sato S: Measures to improve workload of female school-lunch cookers (Abstract in English). J Science of Labour 1988, 64:101-134.

17. Kishi R, Eguchi T, Miyake H: A study of finger deformations, dermatitis and musculo-skeletal symptoms among cooks providing school lunch service (Abstract in English). J Nor Occup Health 1993:10-17.

18. Toyonaga $\mathrm{T}$, Itani $\mathrm{T}$, Takeshita M: Health check for workers in school food supplier: focusing on neck-shoulder-arm pain and low back pain (Abstract in English). JJOMT 200I, 49:478-482

19. Oze Y: Studies on health hazards among cooks providing school lunch service. Report I. The influence of working conditions in central and school kitchens prevalence of health hazards (Abstract in English). Sangyo lgaku 1984, 26:414-424.

20. Oze $Y$ : Studies on health hazards among cooks providing school lunch service. Report 2. An analysis of factors associated with the development of health hazards (Abstract in English). Sangyo Igaku 1984, 26:425-437.

21. Elders LA, Burdorf A: Prevalence, incidence, and recurrence of low back pain in scaffolders during a 3-year follow-up study. Spine 2004, 29:EIOI-106.

22. Lau EM, Egger P, Coggon D, Cooper C, Valenti L, O'Connell D: Low back pain in Hong Kong: prevalence and characteristics compared with Britain. J Epidemiol Commun Health 1995, 49:492-494.

23. Stam HJ, Dommisse AM, Bussmann HJ: Prevalence of low back pain after transfemoral amputation related to physical activity and other prosthesis-related parameters. Disabil Rehabil 2004, 26:794-797.

24. Bejia I, Younes M, Jamila HB, Khalfallah T, Ben Salem K, Touzi M, Akrout $M$, Bergaoui N: Prevalence and factors associated to low back pain among hospital staff. Joint Bone Spine 2005, 72:254-259.

25. Han TS, Schouten JS, Lean ME, Seidell JC: The prevalence of low back pain and associations with body fatness, fat distribution and height. Int J Obes Relat Metab Disord 1997, 21:600-607.
26. Fabunmi AA, Aba SO, Odunaiya NA: Prevalence of low back pain among peasant farmers in a rural community in South West Nigeria. Afr J Med Med Sci 2005, 34:259-262.

27. Bongers PM, de Winter CR, Kompier MA, Hildebrandt VH: Psychosocial factors at work and musculoskeletal disease. Scand J Work Environ Health 1993, 19:297-3 I2.

28. NAASH: School lunch catalogue, 2003. I st edition. Tokyo, Japan: NAASH; 2004.

29. NAASH: School lunch catalogue, 2003. Ist edition. Tokyo, Japan: NAASH; 2004

30. Yip YB, Ho SC, Chan SG: Tall stature, overweight and the prevalence of low back pain in Chinese middle-aged women. Int Obes Relat Metab Disord 200I, 25:887-892.

31. Nyland LJ, Grimmer KA: Is undergraduate physiotherapy study a risk factor for low back pain? A prevalence study of LBP in physiotherapy students. BMC Musculoskelet Disord 2003, 4:22.

32. Kurumatani N, Iki M, Arai T, Moriyama T, Nakachi S, Matsuura $Y$, Nakagiri S: Questionnaire survey of occupational cervicobrachial disorder and low back pain among the cooks providing meals for school children (Abstract in Japanese). JJTOM I987, 35:366-373

33. Leroux I, Brisson C, Montreuil S: Job strain and neck-shoulder symptoms: a prevalence study of women and men white collar workers. Occup Med 2006, 56:102-109.

34. Hopper J, Seeman E: The bone density of female twins discordant for tabacco use. N Engl J Med 1994, 330:387-392.

35. Walsh K, Cruddas M, Coggon D: Low back pain in eight areas of Britain. J Epidemiol Commun Health 1992, 46:227-230.

36. Croft $P$, Rigby A: Socioeconomic influences on back problems in the community Britain. J Epidemiol Commun Health 1994, 48(2): $166-170$.

37. Silman A, Ferry S, Papageorgiou A: Number of children as a risk factor for low back pain in men and women. Arthritis Rheum 1995, 38: 1232-1235.

38. Amano M, Yatsuki K, Umeda G: Cervicobrachial disorders in school-lunch female cooks. (Abstract in Japanese). Sangyo Igaku 1987, 29:366-374.

39. Huang J, Ono Y, Shibata E, Takeuchi Y, Hisanaga N: Occupational musculoskeletal disorders in lunch center workers. Ergonomics 1988, 31:65-75.

40. Yip YB, Ho SC, Chan SG: Identifying risk factors for low back pain (LBP) in Chinese middle-aged women: a case-control study. Health Care Women Int 2004, 25:358-369.

4I. Sakai K, Ito A, Watanabe A, Temmyo Y: Successful experiences in safety and health inspections at school canteens(Abstract in English). J Science Labour 1996, 72:502-504.

42. Lay $A$, Green $C$ : The cost of health and safety in the kitchen. Health Estate ] 1996:4-5.

43. Koda S, Nakagiri S, Yasuda N, Toyota M, Ohara H: A follow-up study of preventive effects on low back pain at worksites by providing a participatory occupational safety and health program. Ind Health 1997, 35:243-242.

44. Kogi K: Participatory methods effective for ergonomic workplace improvement. Appl Ergon 2006, 37:547-554.

\section{Pre-publication history}

The pre-publication history for this paper can be accessed here:

http://www.biomedcentral.com/1471-2458/7/171/pre pub 